\title{
Topical application of ST266 reduces UV-induced skin damage
}

\author{
Linna Guan' \\ Amanda Suggs' \\ Emily Galan' \\ Minh Lam' \\ Elma D Baron ${ }^{1,2}$ \\ 'Department of Dermatology, \\ Case Western Reserve University, \\ ${ }^{2}$ Cleveland Veterans Affairs Medical \\ Center, Cleveland, OH, USA
}

This article was published in the following Dove Press journal:

Clinical, Cosmetic and Investigational Dermatology

10 November 2017

Number of times this article has been viewed
Abstract: Ultraviolet radiation (UVR) has a significant impact on human skin and is the major environmental factor for skin cancer formation. It is also believed that $80 \%$ of the signs of skin aging are attributed to UVR. UVR induces inflammatory changes in the skin via the increase in oxidative stress, DNA damage vascular permeability, and fluctuation in a myriad of cytokines. Acutely, UVR causes skin inflammation and DNA damage, which manifest as sunburn (erythema). ST266 is the secretome of proprietary amnion-derived cells that have been shown to reduce inflammation and accelerate healing of various wounds by promoting migration of keratinocytes and fibroblasts in preclinical animal studies. We hypothesized that ST266 has anti-inflammatory effects that can be used to reduce ultraviolet (UV) erythema and markers of inflammation. In this study, we examined the in vivo effects of ST266 on post UV-irradiated skin by measuring erythema, level of cyclobutane pyrimidine dimer (CPD), and expression level of xeroderma pigmentosum, complementation group A (XPA). We demonstrated that ST266 has the potential to reduce the acute effects of UV-induced skin damage when applied immediately after the initial exposure. In addition, ST266 is shown to reduce erythema, increase XPA DNA repair protein, and decrease damaged DNA.

Keywords: ST266, photoaging, erythema, CPD, XPA, UV-induced DNA damage

\section{Introduction}

Ultraviolet (UV) radiation (UVR) is known to be a tumor initiator and a tumor promoter. ${ }^{1}$ On the skin, UVR can cause neoplasia, atrophy, pigmentary changes, and wrinkling. ${ }^{1}$ UVR is believed to be accountable for up to $80 \%$ of the signs of skin aging. ${ }^{2}$ Morphologically, photoaging comprises cutaneous alterations such as deep wrinkles, roughness, sallowness, mottled dyspigmentation, and telangiectasia along with benign and malignant neoplasms of the skin. ${ }^{3}$ Although effects of long-term UV exposure vary between individuals and are difficult to predict, short-term effects of UVR can be seen almost immediately on the skin as sunburn. ${ }^{2}$ After initial exposure to UVR, the damage to the skin results in an inflammatory process characterized by erythema and DNA damage. ${ }^{4}$ Recovery from UV-induced erythema is a process that requires various cytokines and factors. ${ }^{5}$

ST266 is the secretome of proprietary amnion-derived cells that has been shown to reduce inflammation and accelerate healing of various wounds by promoting migration of keratinocytes and fibroblasts in preclinical animal studies. ${ }^{6-8}$ As a cellular cytokine solution, ST266 is collected by harvesting the proteins released from amnion-derived multipotent progenitor cells. ${ }^{6}$ Cultured cells release various signaling factors that can
Correspondence: Elma D Baron Department of Dermatology, Case Western Reserve University, III 00 Euclid Avenue, Lakeside 3500, Cleveland, $\mathrm{OH} 44106-5028$, USA

$\mathrm{Tel}+\mathrm{I} 216368497 \mid$

$\mathrm{Fax}+12163680212$

Email elma.baron@case.edu 
support or affect the growth, differentiation, and protein production of other cells. ${ }^{6}$

In this study, we investigated the potential of ST266 in the treatment of UV-induced erythema, which is considered as a surrogate marker of DNA damage. To determine the efficacy of ST266, erythema was evaluated at multiple time points post UV irradiation with and without ST266 application. Xeroderma pigmentosum, complementation group A (XPA) expression and cyclobutane pyrimidine dimer (CPD) levels were also evaluated to determine the extent of DNA damage and repair. XPA expression was investigated due to its key role in nucleotide excision repair (NER). Alternatively, CPDs are DNA lesions caused by UVR and are important in evaluating the extent of UV damage. ${ }^{9,10}$

\section{Materials and methods Volunteer selection}

After review and approval by University Hospitals of Cleveland Institutional Review Board, this study was conducted in accordance with the guidelines for good clinical practice and the Declaration of Helsinki (NCT02389777). All subjects were recruited through the Skin Study Center at University Hospitals of Cleveland. In all, 10 totally healthy adults older than 18 years with Fitzpatrick skin type (FST) of I-III were enrolled after they provided written informed consent. ${ }^{11}$ Subjects would have been excluded for history of photodermatosis or photosensitivity disorders, tanning bed use in the last 4 weeks, current intake of photosensitizer drugs (such as doxycycline and thiazide diuretics), current intake of immunosuppressant drugs, or active cancer or cancer within the last 5 years.

\section{Mean erythema dose (MED)}

MED testing was performed similar to a previous study. ${ }^{12}$ First, an eight-holed template with eight $1 \mathrm{~cm}^{2}$ circles was placed on the left buttock skin. The skin was then exposed to increasing doses of simulated solar radiation (SSR). SSR is a full spectrum light source that is used to mimic natural sunlight. SSR was provided by a $1000 \mathrm{~W}$ xenon arc solar simulator model 6271 (Oriel Instruments, Stratford, CT, USA) that emits UV wavelengths from 290 to $400 \mathrm{~nm}$, with a dichroic mirror and 81017bis filter (WG320/1.5 mm). Bentham DM-150 double monochromator spectroradiometer was used to measure the spectrum and integrated irradiance. An IL1700 radiometer (International Light, Newburyport, MA) equipped with a sensor for UVA (SED 033, UVA filter 19672) and UVB (SED 240, UVB filter 15541) positioned 10 inches $(25.4 \mathrm{~cm})$ from the light source was also used to measure irradiance routinely. The amount of time used for irradiation was dependent on the subject's FST (Table 1). This setup is as described in a previous literature. ${ }^{13}$

Visual grading of the erythema was performed after $24 \mathrm{~h}$ to determine the visual MED. Grade " 0 " was given to the area that showed no redness. "Trace" grade was given to an incomplete circle of pink skin. Grade " 1 " was given to a complete circle of pink skin. Grade " 2 " was given to a complete, dark pink to red circle of skin. Skin that showed erythema with the shortest exposure time to SSR was the visual MED. Erythema was further quantified in each skin area that was SSR exposed as well as a nonexposed area three times using the CR-300 chromameter from Konica Minolta (Tokyo, Japan). The difference between the median chromameter reading from the unexposed skin and the median of the SSR-exposed reading is delta $a^{*}$. Following COLIPA recommendations, the minimum erythema dose or MED is defined as the lowest SSR dose that results in a delta $a$ of 2.5 units. This was calculated via linear regression using the log of the SSR exposure times plotted against delta $a^{*}$ values. ${ }^{14}$ Rearranging the $y=m x+b$ equation, $x$ was solved for at $y=2.5$. MED can then be converted to $\mathrm{mJ} / \mathrm{cm}^{2}$ by multiplying the MED in seconds by the UVB irradiance (i.e., dose=irradiance $\times$ time) in seconds.

\section{ST266 treatment}

After determining the MED, a template exposing five 1-inch $(2.5 \mathrm{~cm})$ squares of skin was applied to the right buttock. These sites were numbered 1-5. Sites 1, 2, 3, 4, and 5 were

Table I Eight increasing doses of SSR were given to determine the MED

\begin{tabular}{ll}
\hline SSR times (min and s) & FST \\
\hline $0^{\prime} 2 I^{\prime \prime}$ & I \\
$0^{\prime} 26^{\prime \prime}$ & II \\
$0^{\prime} 32^{\prime \prime}$ & III \\
\hline $0^{\prime} 40^{\prime \prime}$ & IV \\
$0^{\prime} 50^{\prime \prime}$ & \\
$I^{\prime} 02^{\prime \prime}$ & \\
$I^{\prime} 18^{\prime \prime}$ & \\
$I^{\prime} 38^{\prime \prime}$ & \\
$2^{\prime} 02^{\prime \prime}$ & \\
$2^{\prime} 33^{\prime \prime}$ & \\
$3^{\prime} I I^{\prime \prime}$ & \\
\hline $3^{\prime} 59^{\prime \prime}$ & \\
$4^{\prime} 58^{\prime \prime}$ & \\
$6^{\prime} 13^{\prime \prime}$ & \\
\hline
\end{tabular}

Notes: The initial dose of SSR depends on the subject's FST. For example, a subject with FST of III would have received eight doses corresponding with the times boxed in red.

Abbreviations: FST, Fitzpatrick skin type; MED, mean erythema dose; SSR, simulated solar radiation. 
irradiated with two MEDs of SSR, while the rest of the body was draped. ST266 solution (Stemnion, Inc., Pittsburgh, PA, USA) was applied as follows. Site 1 did not receive any ST266 treatment at all. Sites 2 and 3 were treated by investigators with ST266 immediately after irradiation. In all, 8-12 h later or at bedtime, subjects treated Sites 2, 3, 4, and 5 with ST266 at home. ST266 application was also applied by the subjects on Sites 2, 3, 4, and 5 the next morning, $24 \mathrm{~h}$ after SSR. Between 24 and $36 \mathrm{~h}$ after SSR, the subjects returned to the Skin Study Center for Minolta chromameter assessment of erythema at all test sites, as well as for high-resolution digital photography (Canfield, Fairfield, NJ, USA). These assessments were repeated at $48 \mathrm{~h}$ and $72 \mathrm{~h}$ post SSR. Each test site was compared against a nontest site area $(a)$ to determine the difference between the erythema of the test site and the nontest site $(\operatorname{delta} a)$.

\section{Tissue analysis}

In all, $6 \mathrm{~mm}$ punch biopsies were obtained at Site 1 (control) and Site 2 (SSR + immediate ST266 Rx) 24 h post SSR. Nine total samples were analyzed due to inadequate tissue quality from one subject.

Tissue analysis was performed similar to a previous study. ${ }^{12}$ Immunohistochemistry (IHC) was performed using the fixed biopsies that were embedded in paraffin and cut into $5 \mu \mathrm{m}$ sections. Deparaffinization and dehydration were then performed by heating the skin samples in a epitope retrieval buffer at $95-97^{\circ} \mathrm{C}$ for $20 \mathrm{~min}$ and then cooling them for $30 \mathrm{~min}$. The samples were blocked using 5\% normal goat serum in a dilution buffer (Jackson ImmunoResearch Laboratories, Inc., West Grove, PA, USA) and 0.5\% saponin (Sigma-Aldrich Co., St Louis, MO, USA) in $1 \times$ phosphatebuffered saline (PBS). The samples were subsequently incubated for $1 \mathrm{~h}$ at room temperature with polyclonal anti-XPA antibody (Thermo Fisher Scientific, Waltham, MA, USA) or with polyclonal anti-CPD antibody (Cosmo Bio, Tokyo, Japan) in the dilution buffer. The samples were washed in PBS and then incubated with Alexa Fluor 488- or 594-conjugated goat anti-rabbit secondary antibody (Thermo Fisher Scientific) to detect primary antibody as well as Vectashield Mounting Medium for fluorescence with 4',6-diamidino2-phenylindole (DAPI; Vector, Burlingame, CA, USA) to visualize the nucleus. Every experiment included isotype controls to exclude nonspecific antibody staining. Images in this experiment were captured using an UltraVIEW VoX spinning disk confocal system (PerkinElmer Inc., Waltham, MA, USA) mounted on a Leica DMI6000B microscope (Leica Microsystems, Wetzlar, Germany) equipped with an
HC PLAN APO 20×/0.7 objective. Solid-state diode lasers emitting $488 \mathrm{~nm}$ or $561 \mathrm{~nm}$ and $405 \mathrm{~nm}$ excitation light with emission filters were used to capture confocal images of Alexa 488- or 594-conjuated anti-rabbit secondary antibody and DAPI-stained images. MetaMorph Premier Software (Molecular Devices LLC, Sunnyvale, CA, USA) was used quantitatively to analyze the captured images. To quantify fluorescent intensity of the XPA- and CPD-stained samples, the nucleus of the DAPI-stained image was first encircled. The encircled region was then analyzed for fluorescent expression level of each corresponding antibody. MetaMorph was used to measure and record average pixel intensities of these regions. Microsoft Excel was allowed for further analysis of the data. Erythema, XPA, and CPD data were analyzed using paired $t$-test. A difference of $<0.05$ was considered as significant.

\section{Results}

Ten subjects were recruited. Six subjects were males and four females, with ages ranging from 20 to 54 years. Eight subjects were Caucasian and two subjects were Hispanic/ Latino.

\section{Erythema post SSR}

Subjects were irradiated in areas demonstrated in Figure 1. Figure 1 shows three representative subjects $24 \mathrm{~h}$ post SSR exposure who each had a control site (irradiated but not treated with ST266), area treated with immediate ST266, and area treated with ST266 after 8-12 h delay. It can be seen that the erythema is diminished in those treated with immediate ST266 but increased with delayed ST266 application. Primary data for the difference between nonirradiated skin and irradiated skin (delta $\mathrm{a}^{*}$ ) and visual MED grading at $24 \mathrm{~h}, 48 \mathrm{~h}$, and $72 \mathrm{~h}$ are listed in Table S1.

The summary for erythema $\sim 24 \mathrm{~h}$ post SSR exposure for all the subjects, evaluated using delta $a^{*}$, is shown in Figure 2. The mean delta $a^{*}$ for all 10 subject areas corresponding to no ST266 treatment $24 \mathrm{~h}$ post SSR is 9.99, while the mean for all 10 areas with immediate ST266 treatment is 3.74 and the mean for all 10 areas with delayed ST266 treatment $(8-12 \mathrm{~h}$ post SSR) is 13.51. Using a two-tailed paired $t$-test, there was a statistically significant difference between the mean of the areas treated immediately with ST266 and that not treated with ST266 ( $p=0.00079)$. There was also a statistically significant difference between the mean of the areas treated with delayed ST266 and that not treated with ST266 ( $p=0.031)$.

The summary for delta $a^{*} 48 \mathrm{~h}$ post SSR exposure for all the subjects is shown in Figure 3. The mean delta $a^{*}$ for all 10 subjects' areas corresponding to ST266 treatment $48 \mathrm{~h}$ post 


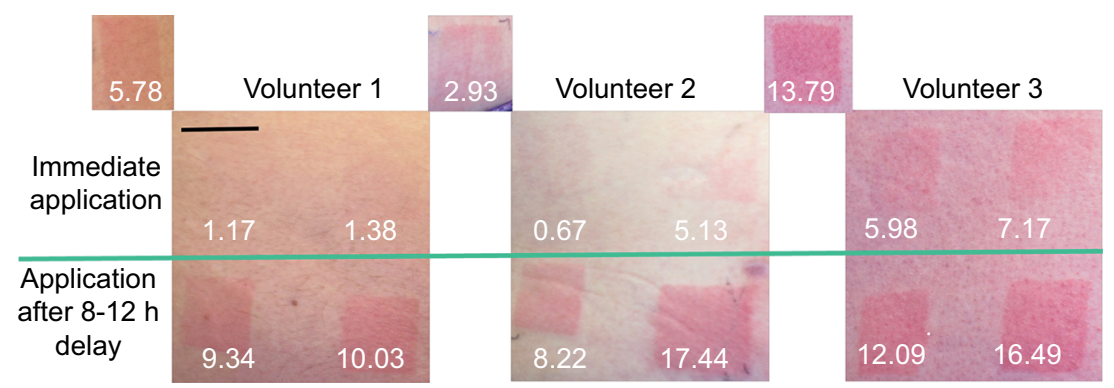

Figure I ST266-treated sites $24 \mathrm{~h}$ after SSR exposure on three representative subjects.

Notes: Control sites are displayed in the top left corner. Delta $a$ values are displayed under each treated site. Delta $a$ is the difference between the erythema of the test site and the nontest site. Black scale bar $=2.54 \mathrm{~cm}$.

Abbreviation: SSR, simulated solar radiation.

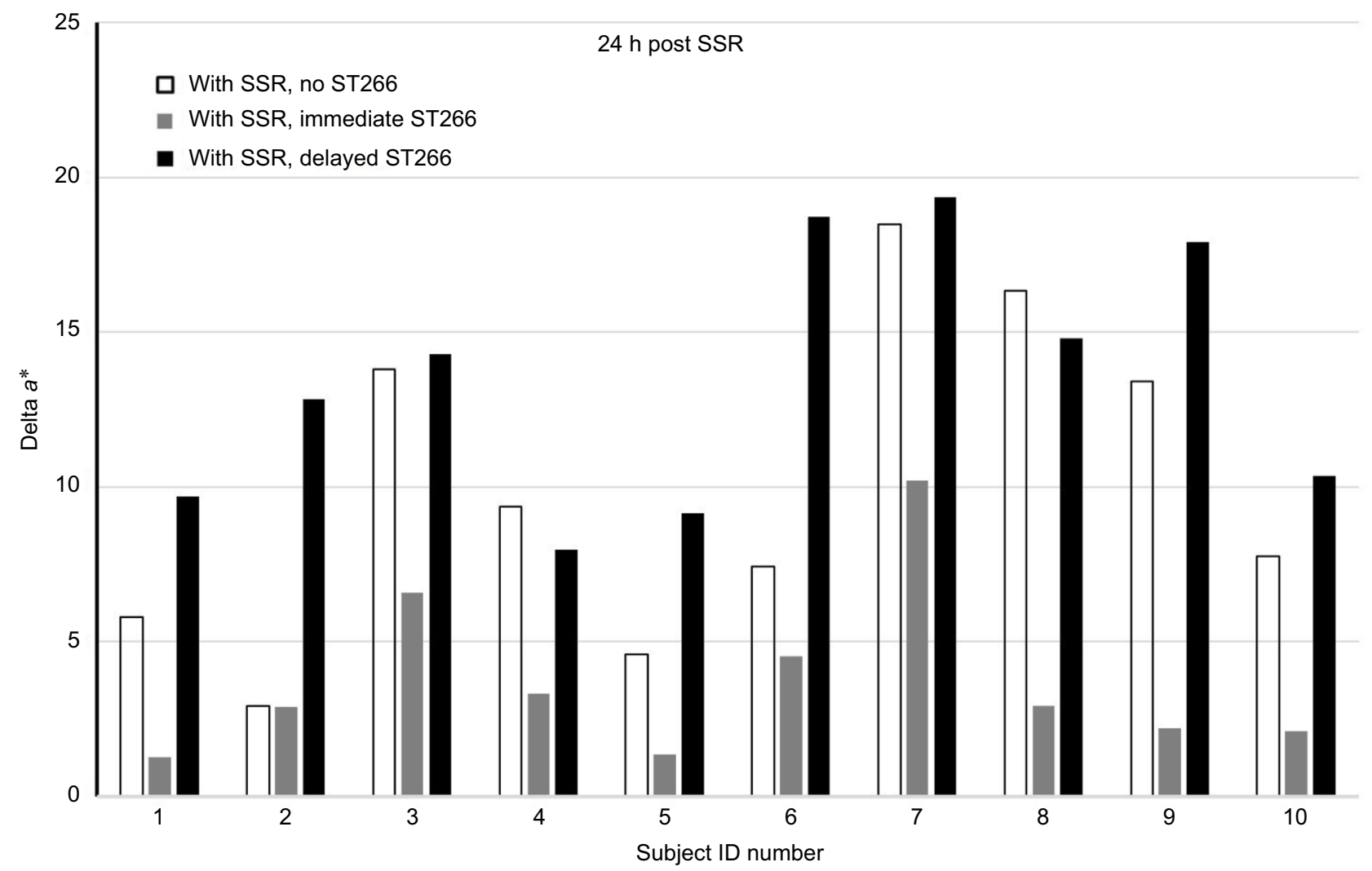

Figure 2 Summary for delta $a^{*} 24 \mathrm{~h}$ post SSR exposure for all of the subjects.

Notes: The mean delta $a^{*}$ for all 10 subject areas corresponding to no ST266 treatment 24 h post SSR is 9.99 , while the mean with immediate ST266 treatment is 3.74 and the mean with delayed ST266 treatment (8-12 h post SSR) is I3.5I. There was a statistically significant difference between the mean of the subject areas treated immediately with ST266 and that not treated with ST266 $(p=0.00079)$ and between the mean of the subject areas treated with delayed ST266 and that not treated with ST266 $(p=0.03 \mathrm{I})$. Abbreviation: SSR, simulated solar radiation.

SSR is 9.62, while the mean with immediate ST266 treatment is 3.56 and the mean with delayed ST266 treatment is 12.09 . There was a statistically significant difference between the mean of the areas treated immediately with ST266 and that not treated with ST266 $(p=0.0070)$. There is a trend between the mean of the areas treated with delayed ST266 and that not treated with ST266 $(p=0.089) 48 \mathrm{~h}$ post SSR.

The summary for delta $a^{*} 72 \mathrm{~h}$ post SSR exposure for all the subjects is shown in Figure 4. The mean delta $a^{*}$ for all
10 subject areas corresponding to no ST266 treatment $72 \mathrm{~h}$ post SSR is 7.56, while the mean with immediate ST266 treatment is 2.97 and the mean with delayed ST266 treatment is 10.64. There was a statistically significant difference between the mean of the areas treated immediately with ST266 and that not treated with ST266 $(p=0.010)$. There is marginal significance between the areas of the subjects treated with delayed ST266 and those not treated with ST266 $(p=0.065)$ $72 \mathrm{~h}$ post SSR. 


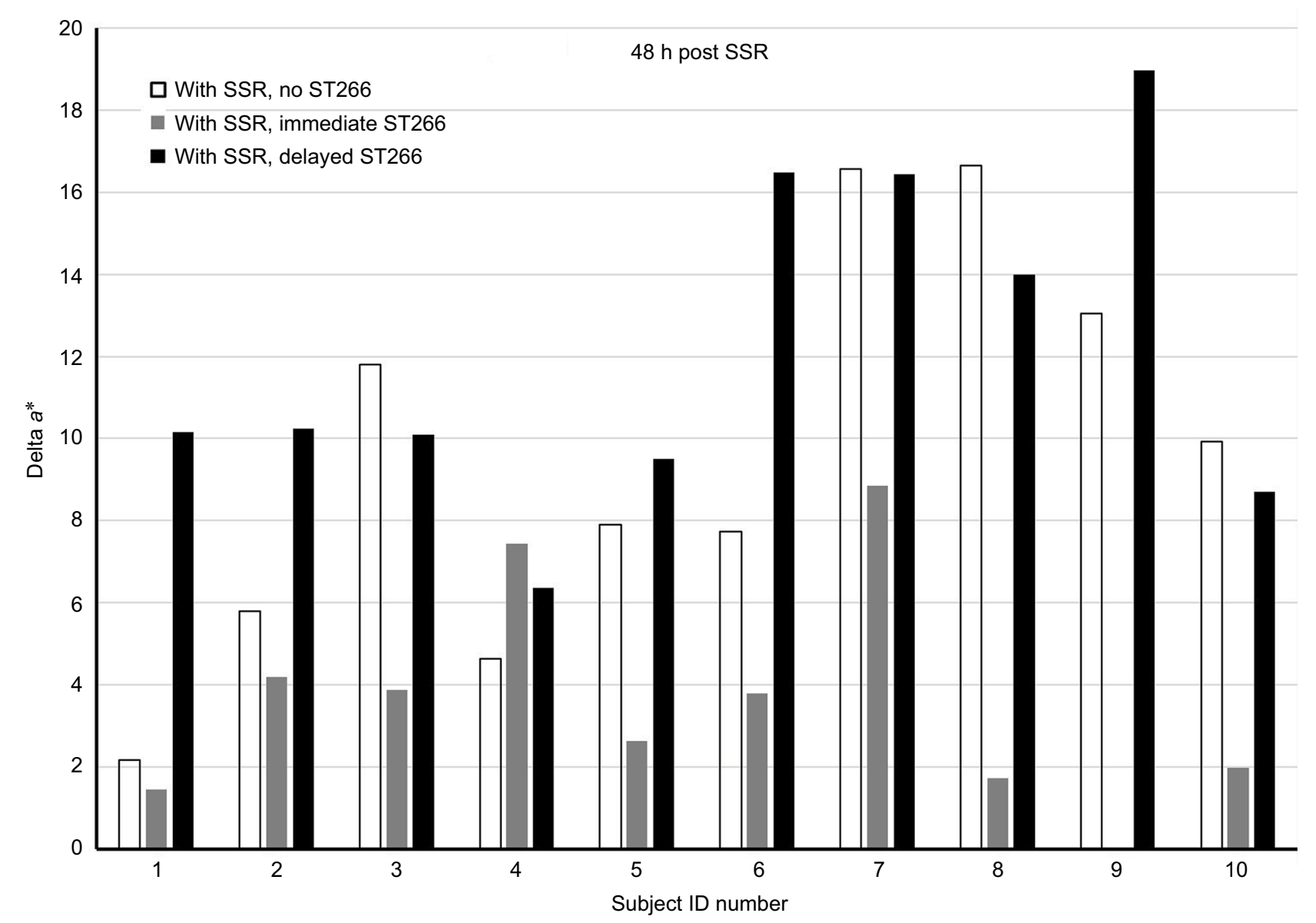

Figure 3 Summary for delta $a^{*} 48 \mathrm{~h}$ post SSR exposure for all the subjects.

Notes: The mean delta $a^{*}$ for all 10 subject areas corresponding to no ST266 treatment $48 \mathrm{~h}$ post SSR is 9.62 , while the mean with immediate ST266 treatment is 3.56 and the mean with delayed ST266 treatment is 12.09 . There was a statistically significant difference between the mean of the subject areas treated immediately with ST266 and that not treated with ST266 $(p=0.0070)$ and a trend between the mean of the subject areas treated with delayed ST266 and that not treated with ST266 ( $p=0.089) 48 \mathrm{~h}$ post SSR.

Abbreviation: SSR, simulated solar radiation.

\section{XPA}

Biopsies from nine out of the 10 subjects were analyzed using anti-XPA antibodies. One subject had insufficient tissue sample for immunostaining. All biopsies were obtained $24 \mathrm{~h}$ post SSR in two areas: 1) without ST266 treatment and 2) immediate ST266 treatment. Figure 5 shows the summary of XPA expression level measured using the mean of pixel fluorescence intensity. Six out of the nine subjects demonstrated higher XPA expression levels with immediate ST266 treatment when compared with no ST266 treatment, while two out of the nine subjects showed lower XPA expression levels with immediate ST266 treatment. One patient demonstrated to have approximately the same XPA expression level with and without ST266 treatment. The mean pixel fluorescence intensity for XPA expression level without ST266 treatment is 4395 arbitrary unit (AU), while the mean XPA expression level with immediate ST266 treatment is 4824 AU. However, two-tailed paired $t$-test was inconclusive with $p=0.55$.

\section{CPD}

Biopsies from eight out of 10 subjects were analyzed using anti-CPD antibodies. The other two subjects' biopsies did not yield sufficient tissue for immunostaining. Figure 6 shows the summary of CPD level measured using the mean of pixel fluorescence intensity. Six out of eight subjects showed lower CPD levels with immediate ST266 treatment when compared with no ST266 treatment $24 \mathrm{~h}$ post SSR. The same subjects that expressed lower XPA levels with ST266 treatment also expressed higher CPD levels. The mean pixel fluorescence intensity for CPD level without ST266 treatment is $4175 \mathrm{AU}$, while the mean CPD level with immediate ST266 treatment is 3209 AU. The difference in CPD levels between no ST266 treatment and immediate ST266 treatment is marginally significant when analyzed using two-tailed paired $t$-test $(p=0.053)$. Interestingly, in all six samples that showed an increase in XPA protein levels in the skin following treatment with ST266, there were fewer CPDs remaining in the skin 


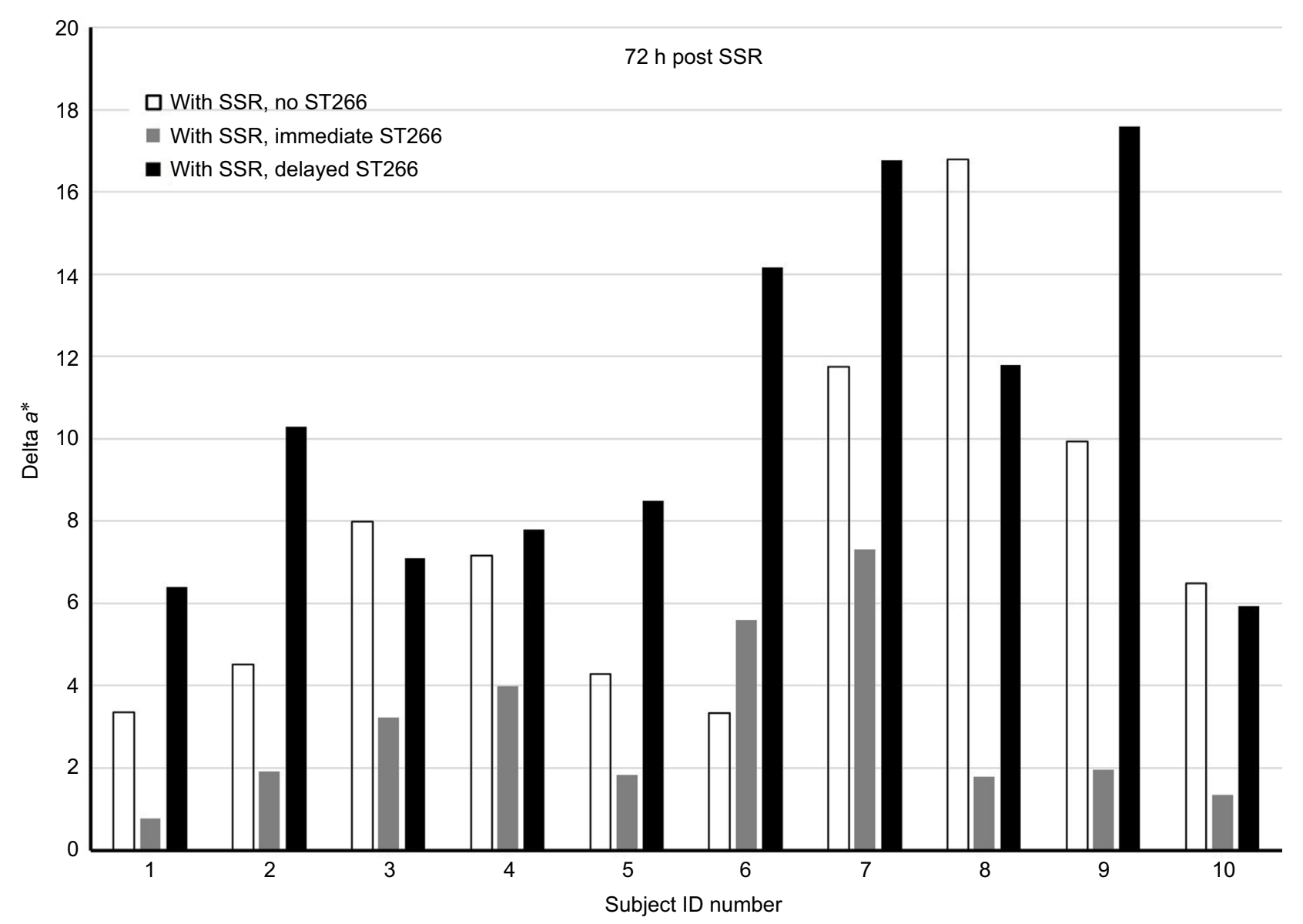

Figure 4 Summary for delta $a^{*} 72 \mathrm{~h}$ post SSR exposure for all the subjects.

Notes: The mean delta $a^{*}$ for all 10 subject areas corresponding to no ST266 treatment 72 h post SSR is 7.56 , while the mean with immediate ST266 treatment is 2.97 and the mean with delayed ST266 treatment is 10.64 . There was a statistically significant difference between the mean of the subject areas treated immediately with ST266 and that not treated with ST266 $(p=0.010)$ and a marginal significance between the mean of the subject areas treated with delayed ST266 and that not treated with ST266 $(p=0.065) 72 \mathrm{~h}$ post SSR.

Abbreviation: SSR, simulated solar radiation.

$24 \mathrm{~h}$ post SSR. In the two samples that demonstrated lower XPA protein levels, there were more CPDs remaining in the skin $24 \mathrm{~h}$ post SSR. There was no association of gender, age, ethnicity, FST, medical history, family history, or medication history between the two subjects who had the reversed CPD and XPA findings.

\section{Discussion}

In the current study, immediate ST266 application was shown to reduce UV-induced erythema and also affect DNA damage and repair pathways. Decrease in DNA damage is likely mediated through the upregulation of DNA repair enzymes such as XPA as demonstrated in Figures 5 and 6 in this study. Although the exact composition of ST266 has not been determined, it has been reported to contain anti-inflammatory cytokines, which could contribute to the decrease in erythema with ST266 treatment when compared to that without ST266 treatment. ${ }^{6}$ Furthermore, ST266 has been shown to contain transforming growth factor- $\beta$ (TGF$\beta){ }^{6}$ One of the major receptors that is downregulated after UV exposure is TGF- $\beta$ type II receptor. ${ }^{15}$ TGF- $\beta$ is a known mediator of procollagen biosynthesis, and dysregulation of TGF- $\beta$ pathway is associated with decreased procollagen biosynthesis. ${ }^{16}$ Whether ST266 also mediates cellular repair via this pathway would be worthwhile to investigate in future studies.

On the other hand, delayed ST266 application of 8-12 h after UV exposure was shown to have the opposite effect. Subjects treated with delayed ST266 showed increase in erythema compared with those not treated with ST266. It is known that signaling factors induced by UV have both proinflammatory as well as anti-inflammatory effects. Some of these key molecules that play dual roles are prostaglandins. ${ }^{17}$ Although data are limited, it has been shown in animal 


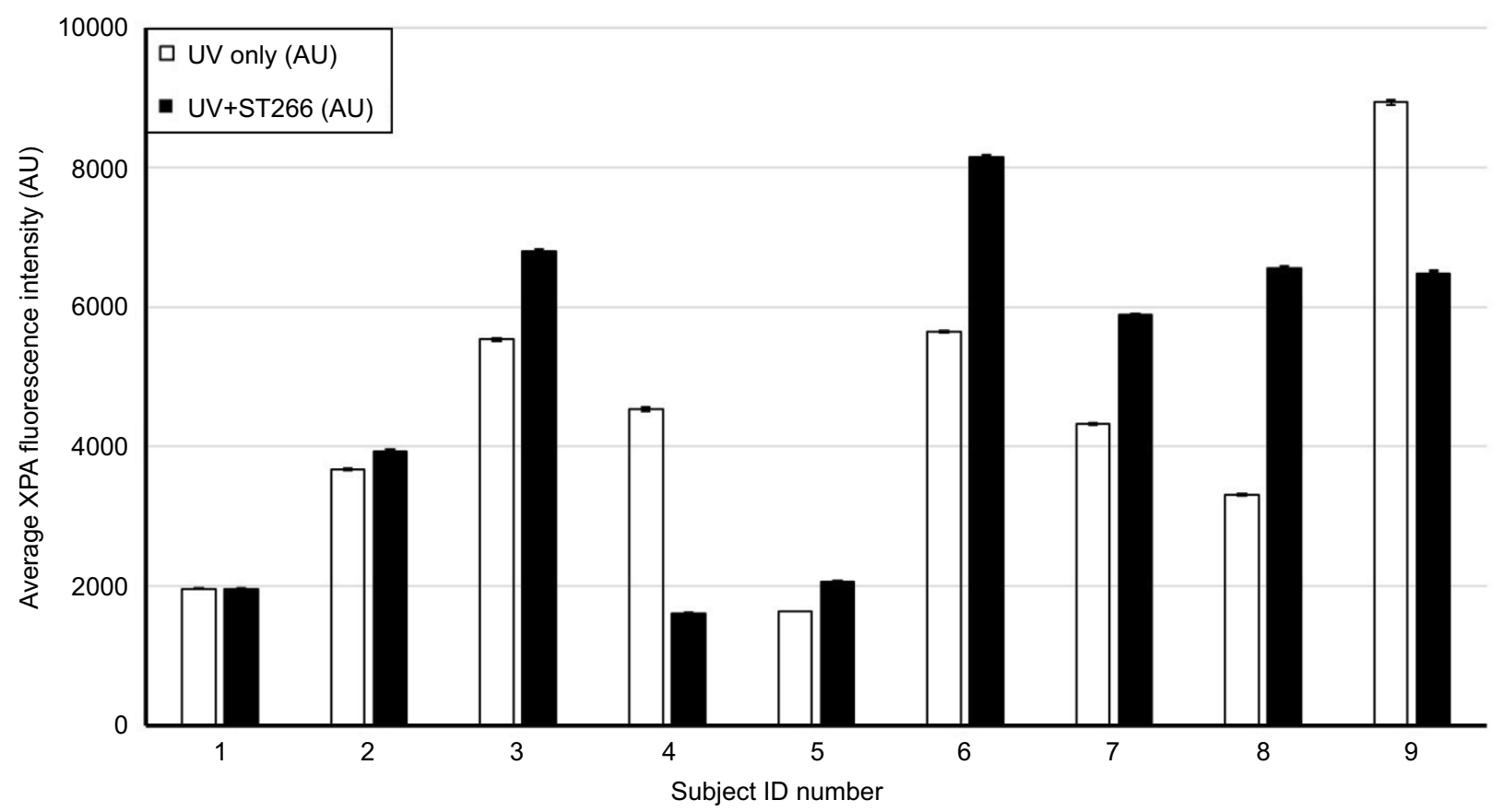

Figure 5 Summary of XPA expression level measured using immunofluorescence.

Notes: Six out of nine subjects showed higher XPA expression level with immediate ST266 treatment when compared with no ST266 treatment. Subjects 4 and 9 demonstrated the opposite with higher XPA expression levels after immediate ST266 treatment. Subject I demonstrated approximately the same XPA expression level with and without ST266 treatment. The mean fluorescence for XPA expression level without ST266 treatment is 4395 AU, while the mean XPA expression level with immediate ST266 treatment is $4824 \mathrm{AU}$. Two-tailed paired $t$-test was unable to demonstrate significance $(p=0.55)$.

Abbreviations: AU, arbitrary unit; UV, ultraviolet; XPA, xeroderma pigmentosum, complementation group $A$.

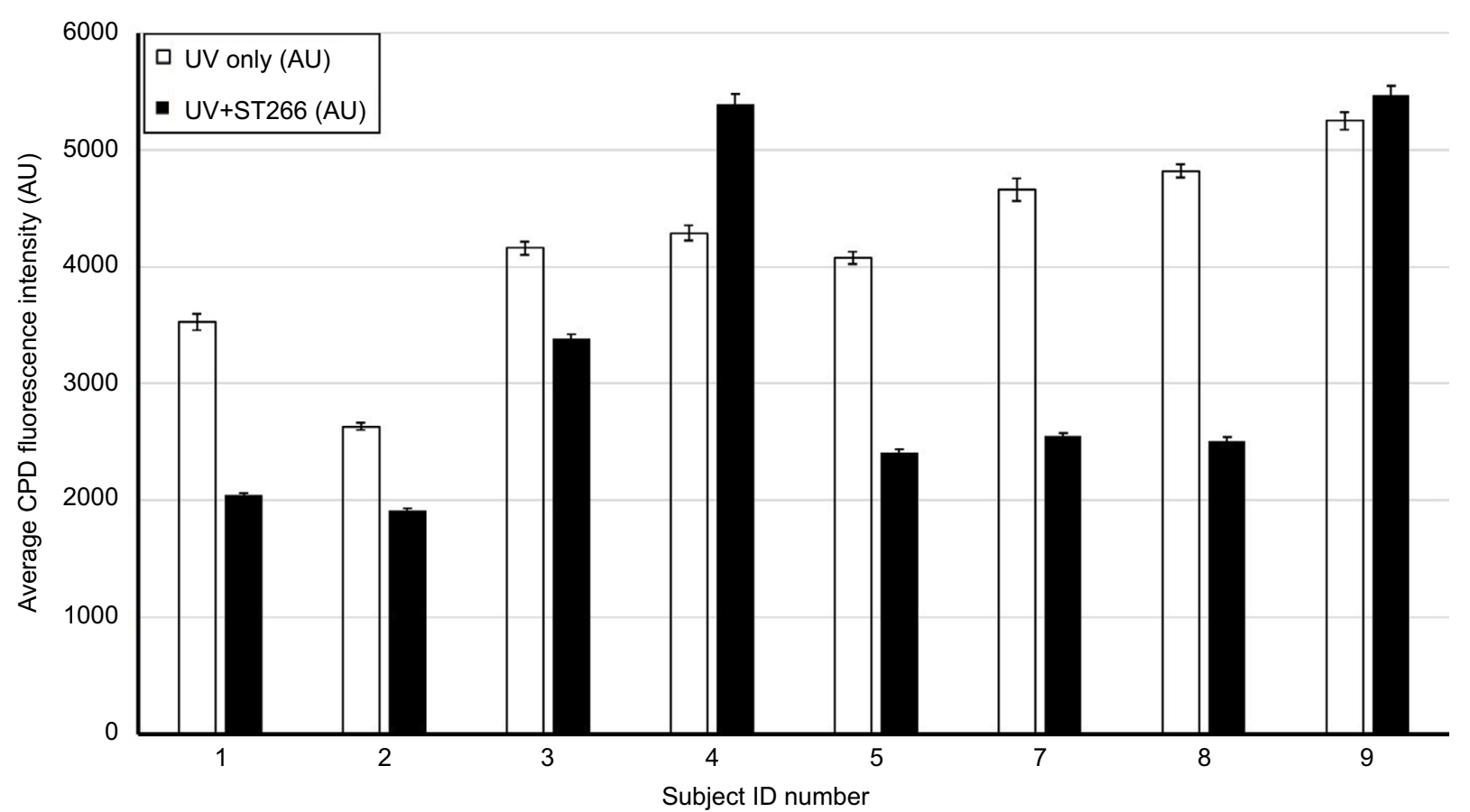

Figure 6 Summary of CPD level measured using immunofluorescence.

Notes: Six out of eight subjects showed lower CPD levels with immediate ST266 treatment when compared with no ST266 treatment 24 h post SSR. Subjects 4 and 9 demonstrated the opposite with higher CPD levels after immediate ST266 treatment. The mean fluorescence intensity for CPD level without ST266 treatment is 4 I 75 AU, while the mean CPD level with immediate ST266 treatment is 3209 AU. The difference in CPD levels between no ST266 treatment and immediate ST266 treatment is marginally significant when analyzed using two-tailed paired $t$-test $(p=0.053)$.

Abbreviations: $\mathrm{AU}$, arbitrary unit; CPD, cyclobutane pyrimidine dimer; SSR, simulated solar radiation; UV, ultraviolet. 
models that synthesis of prostaglandin D and prostaglandin $\mathrm{F}$ declines during acute inflammation but rises again during the resolution of inflammation. ${ }^{17}$ Prostaglandin E2 (PGE2), another proinflammatory lipid mediator, has also been shown to have potent anti-inflammatory effects depending on context. ${ }^{17}$ The anti-inflammatory effect of prostaglandins at a later time point suggests that they may also play a role in the reduction of erythema post SSR. If ST266 contains cytokines that inhibit prostaglandins, application at an early stage can prove to be anti-inflammatory, preventing the prostaglandin activation of inflammation. However, if ST266 application was delayed, inhibition of prostaglandins may produce the opposite effect in preventing resolution of inflammation. Future studies regarding ST266 and prostaglandin expression can further elucidate the mechanism.

Whereas this study focused on short-term biologic outcomes of UV, prevention of harmful UV effects in the long run would be the goal. Chronic inflammation increases mRNAs of various matrix metalloproteinases (MMPs), elastases, and other proteases, which eventually leads to loss of skin tone and resilience, increased roughness and dryness, irregular pigmentation, and deep wrinkles. ${ }^{18,19}$ Skin chronically exposed to UVR become photoaged through promotion of MMP expression, reduction of procollagen synthesis, and connective tissue damage. ${ }^{20}$ Bearing in mind the results in our preliminary study and suggested composition of ST266, we believe that ST266 is a potential tool to investigate not only the prevention of UV-induced erythema but also chronic photoaging. To elucidate some of these properties, further evaluation of changes in molecular markers and using protocols that simulate repeated or long-term UV exposure is necessary.

\section{Acknowledgments}

This study received funding from Stemnion, Inc.

Support for author, Linna Guan, was given by the American Federation for Aging Research (AFAR) through the Medical Student Training In Aging Research (MSTAR) Program.

In addition, this study has been supported in part by the National Institutes of Health (grant 5P30AR039750) via the Skin Diseases Research Center (SDRC) and the Ohio Department of Development, Center for Innovative Immunosuppressive Therapeutics (grant TECH 09023).

The authors thank Dr Scott J Howell for his assistance in image analysis.

The contents of this paper have previously been presented at the following conferences:
1. Guan LL, Suggs A, Galan E, Lam M, Baron E. Topical application of ST266 reduces UV-induced skin damage. Poster presentation: American Geriatric Society 2016 Annual Scientific Meeting; May 19-21, 2016; Long Beach, CA, USA.

2. Galan E, Guan L, Suggs A, Ahsanuddin S, Lam M, Baron ED. Topical application of ST266 reduces UV-induced skin damage. Oral presentation: Society for Investigative Dermatology 2016 Annual Meeting; May 11-14, 2016; Scottsdale, AZ, USA.

\section{Author contributions}

Authors EDB and ML contributed to the design of the study. Authors AS, EG, and EDB conducted the clinical portion of the study. LG and ML performed the basic science portion of the study and analysis of the data. LG, AS, EG, ML, and EDB helped to interpret results. LG drafted the manuscript with help from AS, EG, ML, and EBD. All authors contributed toward data analysis, drafting and critically revising the paper, gave final approval of the version to be published, and agree to be accountable for all aspects of the work.

\section{Disclosure}

The authors report no conflicts of interest in this work.

\section{References}

1. Uitto J. Understanding premature skin aging. N Engl J Med. 1997;337: 1463-1465.

2. Lastowiecka-Moras E, Bugajska J, Młynarczyk B. Occupational exposure to natural $\mathrm{UV}$ radiation and premature skin ageing. Int J Occup Saf Ergon. 2014;20(4):639-645.

3. Gilchrest BA. A review of skin aging and its medical therapy. $\mathrm{Br} J$ Dermatol. 1996;135:867-875.

4. Harrison GI, Young AR. Ultraviolet radiation-induced erythema in human skin. Methods. 2002;28(1):14-19.

5. Nichols JA, Katiyar SK. Skin photoprotection by natural polyphenols: anti-inflammatory, antioxidant and DNA repair mechanisms. Arch Dermatol Res. 2010;302(2):71-83.

6. Steed DL, Trumpower C, Duffy D, et al. Amnion-derived cellular cytokine solution: a physiological combination of cytokines for wound healing. Eplasty. 2008;8:e18.

7. Payne WG, Wachtel TL, Smith CA, Uberti MG, Ko F, Robson MC. Effect of amnion-derived cellular cytokine solution on healing of experimental partial-thickness burns. World J Surg. 2010;34(7): 1663-1668.

8. Uberti MG, Pierpont YN, Ko F, et al. Amnion-derived cellular cytokine solution (ACCS) promotes migration of keratinocytes and fibroblasts. Ann Plast Surg. 2010;64(5):632-635.

9. Matsuda T, Saijo M, Kuraoka I, et al. DNA repair protein XPA binds replication protein A (RPA). J Biol Chem. 1995;270(8):4152-4157.

10. Woollons A, Clingen PH, Price ML, Arlett CF, Green MH. Induction of mutagenic DNA damage in human fibroblasts after exposure to artificial tanning lamps. Br J Dermatol. 1997;137(5):687-692.

11. Marneros AG and Bickers DR, editors. Photosensitivity and Other Reactions to Light. Harrison's Principles of Internal Medicine. 19th ed. Dennis Kasper, et al. New York: McGraw-Hill; 2014. 
12. Guan L, Suggs A, Ahsanuddin S, et al. 2016 arte poster competition first place winner: circadian rhythm and UV-induced skin damage: an in vivo study. J Drugs Dermatol. 2016;15(9):1124-1130.

13. Baron ED, Fourtanier A, Compan D, Medaisko C, Cooper KD, Stevens SR. High ultraviolet A protection affords greater immune protection confirming that ultraviolet A contributes to photoimmunosuppression in humans. J Invest Dermatol. 2003;121(4):869-875.

14. Ferguson J, Brown M, Alert D, et al. Collaborative development of a sun protection factor test method: a proposed European Standard. COLIPA Task Force 'Sun Protection Measurement', Europe. Int J Cosmet Sci. 1996;18(5):203-218.

15. Quan T, He T, Kang S, Voorhees JJ, Fisher GJ. Ultraviolet irradiation alters transforming growth factor beta/smad pathway in human skin in vivo. J Invest Dermatol. 2002;119(2):499-506.
16. $\mathrm{Xu} \mathrm{Y,} \mathrm{Fisher} \mathrm{GJ.} \mathrm{Ultraviolet} \mathrm{(UV)} \mathrm{light} \mathrm{irradiation} \mathrm{induced} \mathrm{sig-}$ nal transduction in skin photoaging. J Dermatol Sci. 2005;1: S1-S8.

17. Scher JU, Pillinger MH. The anti-inflammatory effects of prostaglandins. J Investig Med. 2009;57(6):703-708.

18. Pillai S, Oresajo C, Hayward J. Ultraviolet radiation and skin aging: roles of reactive oxygen species, inflammation and protease activation, and strategies for prevention of inflammation-induced matrix degradation - a review. Int J Cosmet Sci. 2005;27:17-34.

19. Kligman LH, Kligman AM. The nature of photoaging: its prevention and repair. Photodermatol. 1986;3:215-227.

20. Rittié L, Fisher GJ. UV-light-induced signal cascades and skin aging. Ageing Res Rev. 2002;1(4):705-720. 


\section{Supplementary material}

Table SI Summary of raw data for delta $a^{*}$ and visual MED graded at $24 \mathrm{~h}, 48 \mathrm{~h}$, and $72 \mathrm{~h}$

\begin{tabular}{|c|c|c|}
\hline Lesion & Delta $a^{*}$ & Visual \\
\hline \multicolumn{3}{|l|}{ Subject I } \\
\hline \multicolumn{3}{|l|}{$24 \mathrm{~h}$} \\
\hline SSR, no ACCS & 5.78 & $2+$ \\
\hline SSR, immediate ACCS & 1.17 & Trace \\
\hline SSR, immediate ACCS & 1.38 & Trace \\
\hline SSR, delayed ACCS & 9.34 & $2+$ \\
\hline SSR, delayed ACCS & 10.03 & $2+$ \\
\hline \multicolumn{3}{|l|}{ Visit 4} \\
\hline \multicolumn{3}{|l|}{$48 \mathrm{~h}$} \\
\hline SSR, no ACCS & 2.17 & $\mathrm{I}+$ \\
\hline SSR, immediate ACCS & Biopsy & Trace \\
\hline SSR, immediate ACCS & 1.44 & Trace \\
\hline SSR, delayed ACCS & 10.37 & $2+$ \\
\hline SSR, delayed ACCS & 9.94 & $2+$ \\
\hline \multicolumn{3}{|l|}{ Visit 5} \\
\hline \multicolumn{3}{|l|}{$72 \mathrm{~h}$} \\
\hline SSR, no ACCS & 3.36 & $1+$ \\
\hline SSR, immediate ACCS & Biopsy & Trace \\
\hline SSR, immediate ACCS & 0.77 & $\tan$ \\
\hline SSR, delayed ACCS & 4.24 & $2+$ \\
\hline SSR, delayed ACCS & 8.55 & $2+$ \\
\hline \multicolumn{3}{|l|}{ Subject 2} \\
\hline \multicolumn{3}{|l|}{$24 \mathrm{~h}$} \\
\hline SSR, no ACCS & 2.93 & $\mathrm{I}+$ \\
\hline SSR, immediate ACCS & 0.67 & Trace \\
\hline SSR, immediate ACCS & 5.13 & Trace \\
\hline SSR, delayed ACCS & 8.22 & $2+$ \\
\hline SSR, delayed ACCS & 17.44 & $2+$ \\
\hline \multicolumn{3}{|l|}{ Visit 4} \\
\hline \multicolumn{3}{|l|}{$48 \mathrm{~h}$} \\
\hline SSR, no ACCS & 5.79 & $\mathrm{I}+$ \\
\hline SSR, immediate ACCS & Biopsy & Trace \\
\hline SSR, immediate ACCS & 4.19 & Trace \\
\hline SSR, delayed ACCS & 6.10 & $2+$ \\
\hline SSR, delayed ACCS & 14.37 & $2+$ \\
\hline \multicolumn{3}{|l|}{ Visit 5} \\
\hline \multicolumn{3}{|l|}{$72 \mathrm{~h}$} \\
\hline SSR, no ACCS & 4.52 & $1+$ \\
\hline SSR, immediate ACCS & Biopsy & Trace \\
\hline SSR, immediate ACCS & 1.92 & Trace \\
\hline SSR, delayed ACCS & 7.25 & $2+$ \\
\hline SSR, delayed ACCS & 13.34 & $2+$ \\
\hline \multicolumn{3}{|l|}{ Subject 3} \\
\hline \multicolumn{3}{|l|}{$24 \mathrm{~h}$} \\
\hline SSR, no ACCS & 13.79 & $2+$ \\
\hline SSR, immediate ACCS & 5.98 & $1+$ \\
\hline SSR, immediate ACCS & 7.17 & $1+$ \\
\hline SSR, delayed ACCS & 12.09 & $2+$ \\
\hline SSR, delayed ACCS & 16.49 & $2+$ \\
\hline \multicolumn{3}{|l|}{ Visit 4} \\
\hline \multicolumn{3}{|l|}{$48 h$} \\
\hline SSR, no ACCS & 11.80 & $2+$ \\
\hline SSR, immediate ACCS & Biopsy & I+ \\
\hline SSR, immediate ACCS & 3.88 & I+ \\
\hline
\end{tabular}


Table SI (Continued)

\begin{tabular}{|c|c|c|}
\hline Lesion & Delta $a^{*}$ & Visual \\
\hline SSR, delayed ACCS & 11.05 & $2+$ \\
\hline SSR, delayed ACCS & 9.12 & $2+$ \\
\hline \multicolumn{3}{|l|}{ Visit 5} \\
\hline \multicolumn{3}{|l|}{$72 \mathrm{~h}$} \\
\hline SSR, no ACCS & 7.98 & $2+$ \\
\hline SSR, immediate ACCS & Biopsy & I+ \\
\hline SSR, immediate ACCS & 3.23 & $\mathrm{I}+$ \\
\hline SSR, delayed ACCS & 7.70 & $2+$ \\
\hline SSR, delayed ACCS & 6.51 & $2+$ \\
\hline \multicolumn{3}{|l|}{ Subject 4} \\
\hline \multicolumn{3}{|l|}{$24 \mathrm{~h}$} \\
\hline SSR, no ACCS & 9.37 & $2+$ \\
\hline SSR, immediate ACCS & 2.54 & I+ \\
\hline SSR, immediate ACCS & 4.10 & I+ \\
\hline SSR, delayed ACCS & 7.45 & $2+$ \\
\hline SSR, delayed ACCS & 8.51 & $2+$ \\
\hline \multicolumn{3}{|l|}{ Visit 4} \\
\hline \multicolumn{3}{|l|}{$48 \mathrm{~h}$} \\
\hline SSR, no ACCS & 4.64 & $2+$ \\
\hline SSR, immediate ACCS & Biopsy & Biopsy \\
\hline SSR, immediate ACCS & 7.44 & $\mathrm{I}+$ \\
\hline SSR, delayed ACCS & 6.31 & $2+$ \\
\hline SSR, delayed ACCS & 6.40 & $2+$ \\
\hline \multicolumn{3}{|l|}{ Visit 5} \\
\hline \multicolumn{3}{|l|}{$72 \mathrm{~h}$} \\
\hline SSR, no ACCS & 7.17 & $2+$ \\
\hline SSR, immediate ACCS & Biopsy & Biopsy \\
\hline SSR, immediate ACCS & 3.98 & $\mathrm{I}+$ \\
\hline SSR, delayed ACCS & 7.49 & $2+$ \\
\hline SSR, delayed ACCS & 8.12 & $2+$ \\
\hline \multicolumn{3}{|l|}{ Subject 5} \\
\hline \multicolumn{3}{|l|}{$24 \mathrm{~h}$} \\
\hline SSR, no ACCS & 4.59 & $2+$ \\
\hline SSR, immediate ACCS & 0.54 & Trace \\
\hline SSR, immediate ACCS & 2.19 & $\mathrm{I}+$ \\
\hline SSR, delayed ACCS & 5.33 & $2+$ \\
\hline SSR, delayed ACCS & 12.99 & $2+$ \\
\hline \multicolumn{3}{|l|}{ Visit 4} \\
\hline \multicolumn{3}{|l|}{$48 \mathrm{~h}$} \\
\hline SSR, no ACCS & 7.89 & $2+$ \\
\hline SSR, immediate ACCS & Biopsy & Trace \\
\hline SSR, immediate ACCS & 2.63 & Trace \\
\hline SSR, delayed ACCS & 5.17 & $2+$ \\
\hline SSR, delayed ACCS & 13.84 & $2+$ \\
\hline \multicolumn{3}{|l|}{ Visit 5} \\
\hline \multicolumn{3}{|l|}{$72 \mathrm{~h}$} \\
\hline SSR, no ACCS & 4.28 & $\mathrm{I}+$ \\
\hline SSR, immediate ACCS & Biopsy & Trace \\
\hline SSR, immediate ACCS & 1.83 & $\mathrm{I}+$ \\
\hline SSR, delayed ACCS & 4.72 & I+ \\
\hline SSR, delayed ACCS & 12.29 & $2+$ \\
\hline \multicolumn{3}{|l|}{ Subject 6} \\
\hline \multicolumn{3}{|l|}{$24 \mathrm{~h}$} \\
\hline SSR, no ACCS & 7.44 & $2+$ \\
\hline SSR, immediate ACCS & 2.42 & $\mathrm{I}+$ \\
\hline SSR, immediate ACCS & 6.64 & $\mathrm{I}+$ \\
\hline
\end{tabular}


Table SI (Continued)

\begin{tabular}{|c|c|c|}
\hline Lesion & Delta $a^{*}$ & Visual \\
\hline SSR, delayed ACCS & 17.14 & $2+$ \\
\hline SSR, delayed ACCS & 20.33 & $2+$ \\
\hline \multicolumn{3}{|l|}{ Visit 4} \\
\hline \multicolumn{3}{|l|}{$48 \mathrm{~h}$} \\
\hline SSR, no ACCS & 7.73 & $2+$ \\
\hline SSR, immediate ACCS & Biopsy & Trace \\
\hline SSR, immediate ACCS & 3.79 & $\mathrm{I}+$ \\
\hline SSR, delayed ACCS & 12.98 & $2+$ \\
\hline SSR, delayed ACCS & 19.98 & $2+$ \\
\hline \multicolumn{3}{|l|}{ Visit 5} \\
\hline \multicolumn{3}{|l|}{$72 \mathrm{~h}$} \\
\hline SSR, no ACCS & 3.33 & Trace \\
\hline SSR, immediate ACCS & Biopsy & Trace \\
\hline SSR, immediate ACCS & 5.60 & $\mathrm{I}+$ \\
\hline SSR, delayed ACCS & 16.74 & $2+$ \\
\hline SSR, delayed ACCS & 11.57 & $2+$ \\
\hline \multicolumn{3}{|l|}{ Subject 7} \\
\hline \multicolumn{3}{|l|}{$24 \mathrm{~h}$} \\
\hline SSR, no ACCS & 18.48 & $2+$ \\
\hline SSR, immediate ACCS & 11.63 & $2+$ \\
\hline SSR, immediate ACCS & 8.77 & $2+$ \\
\hline SSR, delayed ACCS & 18.19 & $2+$ \\
\hline SSR, delayed ACCS & 20.52 & $2+$ \\
\hline \multicolumn{3}{|l|}{ Visit 4} \\
\hline \multicolumn{3}{|l|}{$48 \mathrm{~h}$} \\
\hline SSR, no ACCS & 16.57 & $\mathrm{I}+$ \\
\hline SSR, immediate ACCS & Biopsy & biopsy \\
\hline SSR, immediate ACCS & 8.85 & $\mathrm{I}+$ \\
\hline SSR, delayed ACCS & 14.66 & $2+$ \\
\hline SSR, delayed ACCS & 18.24 & $2+$ \\
\hline \multicolumn{3}{|l|}{ Visit 5} \\
\hline \multicolumn{3}{|l|}{$72 \mathrm{~h}$} \\
\hline SSR, no ACCS & 11.75 & $2+$ \\
\hline SSR, immediate ACCS & Biopsy & $\mathrm{I}+$ \\
\hline SSR, immediate ACCS & 7.32 & $\mathrm{I}+$ \\
\hline SSR, delayed ACCS & 15.67 & $2+$ \\
\hline SSR, delayed ACCS & 17.87 & $2+$ \\
\hline \multicolumn{3}{|l|}{ Subject 8} \\
\hline \multicolumn{3}{|l|}{$24 \mathrm{~h}$} \\
\hline SSR, no ACCS & 16.34 & $2+$ \\
\hline SSR, immediate ACCS & 3.65 & Trace \\
\hline SSR, immediate ACCS & 2.17 & Trace \\
\hline SSR, delayed ACCS & 12.32 & $2+$ \\
\hline SSR, delayed ACCS & 17.25 & $2+$ \\
\hline \multicolumn{3}{|l|}{ Visit 4} \\
\hline \multicolumn{3}{|l|}{$48 \mathrm{~h}$} \\
\hline SSR, no ACCS & 16.65 & $2+$ \\
\hline SSR, immediate ACCS & Biopsy & Trace \\
\hline SSR, immediate ACCS & 1.73 & Trace \\
\hline SSR, delayed ACCS & 11.91 & $2+$ \\
\hline SSR, delayed ACCS & 16.08 & $2+$ \\
\hline \multicolumn{3}{|l|}{ Visit 5} \\
\hline \multicolumn{3}{|l|}{$72 \mathrm{~h}$} \\
\hline SSR, no ACCS & 16.78 & $2+$ \\
\hline SSR, immediate ACCS & Biopsy & Trace \\
\hline SSR, immediate ACCS & 1.78 & Trace \\
\hline
\end{tabular}


Table SI (Continued)

\begin{tabular}{|c|c|c|}
\hline Lesion & Delta $a^{*}$ & Visual \\
\hline SSR, delayed ACCS & 10.27 & $2+$ \\
\hline SSR, delayed ACCS & 13.32 & $2+$ \\
\hline \multicolumn{3}{|l|}{ Subject 9} \\
\hline \multicolumn{3}{|l|}{$24 \mathrm{~h}$} \\
\hline SSR, no ACCS & 13.40 & $2+$ \\
\hline SSR, immediate ACCS & 1.93 & Trace \\
\hline SSR, immediate ACCS & 2.46 & Trace \\
\hline SSR, delayed ACCS & 16.40 & $2+$ \\
\hline SSR, delayed ACCS & 19.41 & $2+$ \\
\hline \multicolumn{3}{|l|}{ Visit 4} \\
\hline \multicolumn{3}{|l|}{$48 h$} \\
\hline SSR, no ACCS & 13.04 & $2+$ \\
\hline SSR, immediate ACCS & Biopsy & Biopsy \\
\hline SSR, immediate ACCS & -0.31 & Trace \\
\hline SSR, delayed ACCS & 16.60 & $2+$ \\
\hline SSR, delayed ACCS & 21.36 & $2+$ \\
\hline \multicolumn{3}{|l|}{ Visit 5} \\
\hline \multicolumn{3}{|l|}{$72 \mathrm{~h}$} \\
\hline SSR, no ACCS & 9.94 & $2+$ \\
\hline SSR, immediate ACCS & Biopsy & biopsy \\
\hline SSR, immediate ACCS & 1.96 & Trace \\
\hline SSR, delayed ACCS & 14.08 & $2+$ \\
\hline SSR, delayed ACCS & 21.11 & $2+$ \\
\hline \multicolumn{3}{|l|}{ Subject 10} \\
\hline \multicolumn{3}{|l|}{$24 \mathrm{~h}$} \\
\hline SSR, no ACCS & 7.77 & $2+$ \\
\hline SSR, immediate ACCS & 2.31 & Trace \\
\hline SSR, immediate ACCS & 1.93 & Trace \\
\hline SSR, delayed ACCS & 8.03 & $2+$ \\
\hline SSR, delayed ACCS & 12.69 & $2+$ \\
\hline \multicolumn{3}{|l|}{ Visit 4} \\
\hline \multicolumn{3}{|l|}{$48 \mathrm{~h}$} \\
\hline SSR, no ACCS & 9.93 & $2+$ \\
\hline SSR, immediate ACCS & Biopsy & Biopsy \\
\hline SSR, immediate ACCS & 1.98 & $2+$ \\
\hline SSR, delayed ACCS & 9.75 & $2+$ \\
\hline SSR, delayed ACCS & 7.66 & $2+$ \\
\hline \multicolumn{3}{|l|}{ Visit 5} \\
\hline \multicolumn{3}{|l|}{$72 \mathrm{~h}$} \\
\hline SSR, no ACCS & 6.48 & $\mathrm{I}+$ \\
\hline SSR, immediate ACCS & Biopsy & biopsy \\
\hline SSR, immediate ACCS & 1.35 & Trace \\
\hline SSR, delayed ACCS & 5.59 & $\mathrm{I}+$ \\
\hline SSR, delayed ACCS & 6.27 & $\mathrm{I}+$ \\
\hline
\end{tabular}

Note: Skin that could not be evaluated due to biopsies was noted with "Biopsy".

Abbreviations: ACCS, amnion-derived cellular cytokine solution; MED, mean erythema dose; SSR, simulated solar radiation.

Clinical, Cosmetic and Investigational Dermatology

\section{Publish your work in this journal}

Clinical, Cosmetic and Investigational Dermatology is an international, peer-reviewed, open access, online journal that focuses on the latest clinical and experimental research in all aspects of skin disease and cosmetic interventions. This journal is included on PubMed. The manuscript management system is completely online and includes a very quick and fair peer-review system, which is all easy to use. Visit http://www.dovepress.com/testimonials.php to read real quotes from published authors 